\title{
Low hepatic stearoyl-CoA desaturase 1 activity is associated with fatty liver and insulin resistance in obese humans
}

\author{
N. Stefan - A. Peter - A. Cegan • H. Staiger • \\ J. Machann - F. Schick • C. D. Claussen • A. Fritsche • \\ H.-U. Häring • E. Schleicher
}

Received: 23 October 2007 / Accepted: 2 January 2008 / Published online: 20 February 2008

(C) Springer-Verlag 2008

\begin{abstract}
Aims/hypothesis Stearoyl-CoA desaturase 1 (SCD1) is the rate-limiting enzyme in monounsaturated fatty acid synthesis. It is imperative for the assembly of VLDL particles, which transport triacylglycerol (TG) from liver to adipose tissue and other sites. We aimed to determine the role of hepatic SCD1 activity in human glucose and lipid metabolism.

Methods We studied 54 people participating in a lifestyle intervention programme with diet modification and increased physical activity. Insulin sensitivity was determined during a euglycaemic-hyperinsulinaemic clamp and estimated from an OGTT. Liver fat was quantified by ${ }^{1} \mathrm{H}$-magnetic resonance spectroscopy at baseline and after 9 months of intervention. The pattern of fatty acids in serum VLDL-TGs was determined by ultracentrifugation followed by thin layer and gas chromatography, with the 18:1 n-9: 18:0 ratio providing an index of hepatic SCD1 activity.

Results The hepatic SCD1 activity index correlated negatively with liver fat $(r=-0.29, p=0.04)$ and positively with insulin sensitivity, both OGTT-derived $(r=0.42, p=0.003)$
\end{abstract}

N. Stefan and A. Peter contributed equally to this work.

N. Stefan $(\varangle) \cdot$ A. Peter $\cdot$ A. Cegan $\cdot$ H. Staiger $\cdot$ A. Fritsche H.-U. Häring • E. Schleicher

Department of Internal Medicine, Division of Endocrinology, Diabetology, Vascular Medicine,

Nephrology and Clinical Chemistry, University of Tübingen, Otfried-Müller-Str. 10,

72076 Tübingen, Germany

e-mail: norbert.stefan@med.uni-tuebingen.de

J. Machann $\cdot$ F. Schick $\cdot$ C. D. Claussen

Section of Experimental Radiology, University of Tübingen,

Tübingen, Germany and clamp-derived $(r=0.27, p=0.07)$. These correlations depended on overall adiposity. They were absent in leaner participants $(n=27$, liver fat: $p=0.34$, insulin sensitivity [OGTT]: $p=0.75$, insulin sensitivity [clamp]: $p=0.24$ ), but were strong in obese individuals $(n=27, p=0.004, p=0.0002$ and $p=0.006$, respectively). Furthermore, during intervention a high SCD1 activity index at baseline predicted a decrease in liver fat only in obese participants $(r=-0.46, p=0.02)$. Conclusions/interpretation Our data suggest that high hepatic SCD1 activity may regulate fat accumulation in the liver and possibly protects from insulin resistance in obesity.

Keywords Diabetes $\cdot$ Fatty acid pattern $\cdot$ Fatty liver $\cdot$ Insulin resistance - Obesity · Prevention - Proton magnetic resonance spectroscopy · Stearoyl-CoA desaturase 1 . Thin layer chromatography . Very low density lipoprotein

Abbreviations
$\begin{array}{ll}{ }^{1} \text { HMRS } & \text { proton magnetic resonance spectroscopy } \\ \text { NAFLD } & \text { non-alcoholic fatty liver disease } \\ \text { SCD1 } & \text { stearoyl-CoA desaturase 1 } \\ \text { TG } & \text { triacylglycerol } \\ \text { TLC } & \text { thin layer chromatography }\end{array}$

Introduction

Non-alcoholic fatty liver disease (NAFLD) is the main cause of elevated serum liver enzymes among the general population and generates a wide spectrum of liver damage ranging from simple steatosis to steatohepatitis, advanced fibrosis and cirrhosis with end-stage liver disease [1]. NAFLD predicts development of the metabolic syndrome and is involved in the pathophysiology of atherosclerosis, 
insulin resistance and type 2 diabetes [1-7]. While these data emphasise the critical role of fatty liver in human metabolism, the mechanisms involved in the process of hepatic fat accumulation in humans are not fully understood. Circulating levels of adiponectin, the adipokine that increases lipid oxidation in liver and skeletal muscle $[8,9]$, together with genetic variability in the adiponectin receptor genes [10], as well as in the upstream transcription factor 1 and hepatic lipase genes [11], all of which regulate lipolysis and lipid metabolism, may affect hepatic fat accumulation. Furthermore, a high intake of total fat $[12,13]$ and particularly of saturated fat [14], as well as the amount and the pattern of fatty acids released from adipocytes to the liver [12, 15], may also be involved. However, fatty acids also derive from de novo lipogenesis in the liver [12, $15,16]$, which is predominantly regulated by sterolregulatory binding protein 1c [17]. After the assembly of triacylglycerol (TG), VLDL particles transport fatty acids from the liver to adipose tissue and other sites [17]. The enzyme stearoyl-CoA desaturase 1 (SCD1), which is predominantly expressed in liver but also in a variety of other tissues, plays an important role in this pathway. SCD1 is a microsomal enzyme that catalyses the synthesis of monounsaturated long-chain fatty acids from saturated fatty acyl-CoAs. The preferred substrates for SCD1 are palmitoyl(16:0) and stearoyl-CoA (18:0), which are converted to palmitoleoyl- (16:1 n-7) and oleoyl- (18:1 n-9) CoA respectively. These fatty acids in the ester form are the major constituents of VLDL-TGs [18]. The saturated/monounsaturated fatty acid ratio modulates the composition of phospholipids in cell membranes and affects membrane fluidity; differences in this ratio are associated with a number of diseases, including diabetes and obesity [19-23].

Mice with whole-body SCD1 deficiency were lean [24] and had decreased liver fat content [25, 26], suggesting that lack of SCD1 protects from fat accumulation in the liver. However, these lean SCD1-deficient mice developed severe hypercholesterolaemia, had a large decrease in HDLcholesterol levels and displayed lower VLDL production than the wild-type on a low-fat, high-carbohydrate diet [27]. This finding implies that liver-specific downregulation of SCD1 may impair VLDL assembly and thus may abolish the capacity of the liver to clear intrahepatic TG. Accordingly, liver-specific downregulation of SCD1 resulted in an increase, not a decrease in liver fat in rodents [28]. The latter findings suggest that total SCD1 deficiency represents a relatively extreme phenotype that may not easily be compared with human physiology. This is supported by a recent finding that hepatic-specific SCD1 deficiency does not protect from high-fat diet-induced hepatic steatosis or insulin resistance [29].

To our knowledge, there are no data on the relationships of hepatic SCD1 activity with glucose and lipid metabolism in humans. In the present study, we specifically hypothesised that hepatic SCD1 activity may be involved in the pathophysiology of fatty liver and insulin resistance in humans. Therefore, we determined the 18:1 n-9: 18:0 ratio in the serum VLDL-TGs that are assembled in the liver, to estimate hepatic SCD1 activity and tested the relationships of this index with liver fat, as measured by ${ }^{1} \mathrm{H}$-magnetic resonance spectroscopy ( ${ }^{1}$ HMRS), and with insulin sensitivity.

\section{Methods}

\section{Participants}

A total of 54 individuals were included in the present study. They were participating in an ongoing trial to reduce adiposity and to prevent type 2 diabetes [10]. Individuals were recruited from the southern part of Germany. They were randomly included in this study on condition that they fulfilled at least one of the following criteria: a family history of type 2 diabetes, a BMI $>27 \mathrm{~kg} / \mathrm{m}^{2}$, previous diagnosis of impaired glucose tolerance or gestational diabetes. The participants were not taking any medication known to affect glucose tolerance or insulin sensitivity. None of them had a history of liver disease or regularly consumed alcohol. They were considered healthy according to a physical examination and routine laboratory tests. Most of them were sedentary and not involved in regular exercise programmes. Participants underwent baseline measurements and had data re-measured at 9 months of follow-up after they had undergone dietary counselling and increased physical activity. Counselling aimed to reduce body weight by $\geq 5 \%$ and total intake of energy (particularly from fat) to $<30 \%$ of energy consumed, and at increasing intake of fibre to at least $15 \mathrm{~g} / 4187 \mathrm{~kJ}(1,000 \mathrm{kcal})$. Furthermore, they were asked to reduce intake of saturated fat to $\leq 10 \%$ of energy. Participants were instructed to increase physical activity and to perform at least $3 \mathrm{~h}$ of moderate sports per week. For the day prior to tests, participants were instructed to refrain from heavy exercise and retain their regular diets, mostly consisting of a Western diet at baseline and the aforementioned healthy diet at follow-up. Informed written consent was obtained from all participants and the local medical ethics committee approved the protocol.

Body composition and body fat distribution

Body fat was measured by the bioelectrical impedance method (RJL, Detroit, MI, USA). Visceral fat was measured with an axial T1-weighted fast spin echo technique with a $1.5 \mathrm{~T}$ whole-body imager (Magnetom Sonata; Siemens Medical Solutions, Erlangen, Germany) [30]. 
${ }^{1} \mathrm{HMRS}$ for quantitative analysis of liver fat

Liver fat was measured by localised ${ }^{1} \mathrm{HMRS}$ as previously described [10]. We chose a liver fat cut-off of 5.5\% as the threshold to denote steatosis. This cut-off has been identified to separate participants with fatty liver from healthy control persons [31].

Oral glucose tolerance test

All individuals underwent a $75 \mathrm{~g}$ OGTT. We obtained venous plasma samples at $0,30,60,90$ and $120 \mathrm{~min}$ for determination of plasma glucose and insulin. Glucose tolerance was determined according to the 1997 WHO diagnostic criteria [32]. Insulin sensitivity was calculated from glucose and insulin values during the OGTT, as proposed by Matsuda and DeFronzo [33].

Euglycaemic-hyperinsulinaemic clamp

A total of 48 participants underwent measurements of insulin sensitivity by the euglycaemic-hyperinsulinaemic clamp. At about 07:00 hours, after a $12 \mathrm{~h}$ overnight fast, an antecubital vein was cannulated for infusion of insulin and glucose. A dorsal hand vein of the contralateral arm was cannulated and placed under a heating device to permit sampling of arterialised serum. After basal blood was drawn, participants received a primed insulin infusion at a rate of $40 \mathrm{mU} \mathrm{m}^{-2} \mathrm{~min}^{-1}$ for $2 \mathrm{~h}$. Blood was drawn every $5 \mathrm{~min}$ for determination of serum glucose. Glucose infusion was adjusted appropriately to maintain the fasting glucose level. Insulin sensitivity $\left(\mu \mathrm{mol} \mathrm{kg}{ }^{-1} \min ^{-1}[\mathrm{pmol} / 1]^{-1}\right)$ for systemic glucose uptake was calculated as the mean infusion rate of glucose ( $\mu \mathrm{mol} \mathrm{kg}{ }^{-1} \mathrm{~min}^{-1}$ ) necessary to maintain euglycaemia during the last $60 \mathrm{~min}$ of the euglycaemic-hyperinsulinaemic clamp divided by the steady-state plasma insulin concentration.

Analytical procedures

Plasma and serum glucose, insulin, NEFA and lipids Serum glucose was determined using a bedside glucose analyser (glucose-oxidase method; YSI, Yellow Springs Instruments, Yellow Springs, CO, USA). For measurement of insulin and total NEFA and lipoproteins, blood was placed on ice after drawing, immediately transferred to the laboratory and subsequently analysed. Plasma insulin was determined by microparticle enzyme immunoassay (ADVIA Centaur; Bayer HealthCare, Fernwald, Germany) and total serum NEFA concentrations were measured with an enzymatic method (WAKO Chemicals, Neuss, Germany). Serum total cholesterol and HDL- and LDL-cholesterol concentrations were measured with a standard colorimetric method using the Bayer analyser (Bayer HealthCare).
Fatty acid pattern Fasting plasma samples were spun down, frozen immediately and stored at $-80^{\circ} \mathrm{C}$ for analysis of the fatty acid pattern. First, the plasma VLDL-fraction was separated from the HDL- and LDL-cholesterol fraction by ultracentrifugation. For this, $1.5 \mathrm{ml}$ sodium chloride solution $(1.006 \mathrm{~g} / \mathrm{ml})$ was added to $1.5 \mathrm{ml}$ human plasma. Subsequently, ultracentrifugation at 40,000 rpm $(817,480 \mathrm{~g})$ and $10^{\circ} \mathrm{C}$ was done for $18 \mathrm{~h}$ using a preparative ultracentrifuge (Optima; Beckman Coulter, Palo Alto, CA, USA). Afterwards, the top layer (VLDL fraction) was removed and used for further analyses.

Next, the VLDL fraction was divided, using thin layer chromatography (TLC), into five subfractions containing phospholipids, diacylglycerol, NEFA, TG and cholesteryl esters. In detail, the VLDL-fraction was cleared from protein using 2-propanol, $n$-heptane and $2 \mathrm{~mol} / \mathrm{l}$ phosphoric acid. Toluol, methanol and water were added, and after centrifugation at 4,000 rpm $(8,175 \mathrm{~g})$ the upper phase was dried under nitrogen. The lipids were solved in $\mathrm{CHCl}_{3}-\mathrm{CH}_{3} \mathrm{OH}$ and applied to a silica gel chromatography plate (Merk, Darmstadt, Germany). The phospholipids, diacylglycerol, NEFA, TG and cholesteryl esters were separated using $n$-hexane, diethylether and acetic acid as a solvent. To identify the localisation of the fractions, a pooled control plasma was also separated on each plate and visualised by 2,7-dichlor-fluoresceine under ultraviolet light. The TG fraction was scraped off the TLC plate, transferred to screw-capped vials and dissolved in methanol/toluol mixture (4:1, vol./vol.) containing cis-13,16,19-docosatrienoic acid as an internal standard. Trans-esterification was performed by incubation with acetyl chloride at $100^{\circ} \mathrm{C}$. The cold sample was neutralised with $\mathrm{K}_{2} \mathrm{CO}_{3}$ and the upper phase was concentrated to $80 \mu \mathrm{l}$ under nitrogen. The fatty acid methyl esters were quantified with cis-13,16,19-docosatrienoic acid as the internal standard using gas chromatography with a flame ionisation detector, as previously described [34]. In a subgroup of 38 participants for whom a sufficient sample volume was available, the fatty acid pattern was also determined in the NEFA fraction in serum.

Calculation of the desaturase activity indexes As 18:0 but not 16:0 is the preferred substrate of SCD1 in humans [35], the 18:1 n-9: 18:0 ratio was calculated as an index of hepatic SCD1 $(\Delta 9)$ desaturase activity. Furthermore, the 20:4 $n-6: 20: 3 n-6$ and 18:3n-6: 18:2 n-6 ratios were calculated as indexes of hepatic $\Delta 5$ and $\Delta 6$ desaturase activities, respectively.

Statistical analyses

Data are given as mean $\pm \mathrm{SE}$. Statistical comparison between variables was performed using logarithmically transformed data (for non-normally distributed parameters). 
Differences between baseline and follow-up were tested using the matched pairs $t$ test. To adjust for the effects of relevant covariates (age, sex, percentage of body fat), multivariate linear regression analyses were performed. A $p$ value $\leq 0.05$ was considered statistically significant. The statistical software package JMP 4.0 (SAS Institute, Cary, $\mathrm{NC}$, USA) was used.

\section{Results}

Baseline data

Characteristics of the population are shown in Table 1. The two sexes were evenly represented. Anthropometrics and metabolic characteristics covered a wide range that was particularly large for body fat, liver fat and insulin sensitivity.

At baseline, the hepatic SCD1 activity index was not different between males $(11.2 \pm 0.7)$ and females $(9.8 \pm 0.7$, $p=0.18$ ) and was not correlated with age or total body fat ( $p \geq 0.59$ for all). The hepatic SCD1 activity index was also not correlated with visceral fat $(p=0.48)$. However, it was negatively correlated with liver fat $(r=-0.29, p=0.04)$, when adjusted for sex and body fat in multivariate regression analyses. In addition, participants with fatty liver $(n=21$, liver fat $>5.5 \%$ ) had a lower hepatic SCD1 activity index $(9.5 \pm 0.8)$ than healthy control participants $(n=33,11.0 \pm$ $0.7)$, independently of sex and body fat $(p=0.04)$.

In univariate analyses, sex and body fat were determinants of metabolic parameters. Regarding the relationships of the hepatic SCD1 activity index, adjusted for sex and body fat in multivariate linear regression analyses, with metabolic parameters, no correlations with fasting glycaemia and NEFA were observed (both $p \geq 0.43$ ). However, the adjusted hepatic SCD1 activity index was negatively correlated with fasting insulinaemia $(r=-0.28, p=0.04)$, as well as with $2 \mathrm{~h}$ glycaemia $(r=-0.30, p=0.03)$ and $2 \mathrm{~h}$ insulinaemia $(r=-0.41, p=0.002)$. In addition, the adjusted hepatic SCD1 activity index was positively correlated with insulin sensitivity estimated from the OGTT $(r=0.42, p=0.003)$. A statistical trend was seen for a positive correlation with insulin sensitivity determined during the clamp $(r=0.27$, $p=0.07)$. Furthermore, the 16:1 n-7: 16:0 ratio also correlated positively with insulin sensitivity by OGTT $(r=$ $0.31, p=0.02$ ).

Because low insulin sensitivity is associated with a low activity of $\Delta 5$ and a high activity of $\Delta 6$ desaturases as calculated from product/precursor ratios in muscle and adipose tissue [36], we also investigated whether indices

Table 1 Participant characteristics at baseline and after 9 months of follow-up

\begin{tabular}{|c|c|c|c|}
\hline & Baseline & Follow-up & $p$ value $^{\mathrm{a}}$ \\
\hline \multicolumn{4}{|l|}{ Demographics and body composition } \\
\hline Sex, men/women $(n)$ & $26 / 28$ & & - \\
\hline Age (years) & $44 \pm 2(23-65)$ & $45 \pm 2(24-66)$ & - \\
\hline Body weight $(\mathrm{kg})$ & $86.5 \pm 2.2(53.7-123.0)$ & $83.9 \pm 2.1(55.8-114.4)$ & $<0.0001$ \\
\hline BMI $(\mathrm{kg} / \mathrm{m})$ & $29.3 \pm 0.6(19.4-40.2)$ & $28.4 \pm 0.6(18.6-38.1)$ & $<0.0001$ \\
\hline Body fat ${ }_{\text {bioimpedance }}(\%)$ & $31.0 \pm 1.0(16.0-50.0)$ & $30.1 \pm 1.2(7.3-54.9)$ & 0.06 \\
\hline Visceral fat ${ }_{M R T}(\mathrm{~kg})$ & $3.3 \pm 0.2(0.5-7.6)$ & $2.7 \pm 0.2(0.3-6.4)$ & $<0.0001$ \\
\hline Liver fat $_{1 \text { HMRS }}(\%)$ & $6.3 \pm 0.8(0.5-29.1)$ & $4.3 \pm 0.6(0.1-18.1)$ & $<0.0001$ \\
\hline \multicolumn{4}{|l|}{ Metabolic characteristics } \\
\hline Fasting glucose (mmol/l) & $5.15 \pm 0.06(4.22-5.89)$ & $5.07 \pm 0.6(4.28-6.39)$ & 0.05 \\
\hline $2 \mathrm{~h}$ glucose $(\mathrm{mmol} / \mathrm{l})$ & $6.81 \pm 0.22(4.17-11.0)$ & $6.40 \pm 0.22(3.50-9.94)$ & 0.02 \\
\hline Fasting insulin (pmol/1) & $57 \pm 4(19-135)$ & $50 \pm 4(14-148)$ & 0.007 \\
\hline $2 \mathrm{~h}$ insulin $(\mathrm{pmol} / \mathrm{l})$ & $475 \pm 49(78-1513)$ & $412 \pm 57(50-1913)$ & 0.01 \\
\hline Fasting serum NEFA $(\mu \mathrm{mol} / \mathrm{l})$ & $588 \pm 33(199-1291)$ & $627 \pm 27(199-1188)$ & 0.94 \\
\hline Fasting TG $(\mathrm{mmol} / \mathrm{l})$ & $1.82 \pm 0.23(0.57-11.40)$ & $1.25 \pm 0.10(0.44-4.33)$ & 0.008 \\
\hline Fasting total cholesterol $(\mathrm{mmol} / \mathrm{l})$ & $4.84 \pm 0.10(2.50-6.29)$ & $4.60 \pm 0.10(2.39-6.14)$ & 0.0004 \\
\hline Fasting HDL-cholesterol (mmol/1) & $1.32 \pm 0.05(0.81-2.39)$ & $1.31 \pm 0.05(0.81-2.55)$ & 0.48 \\
\hline Fasting LDL-cholesterol (mmol/l) & $3.07 \pm 0.08(1.04-4.50)$ & $2.94 \pm 0.70(1.04-4.76)$ & 0.03 \\
\hline $\mathrm{IS}_{\text {clamp }}\left(\mu \mathrm{mol} \mathrm{kg}{ }^{-1} \min ^{-1}[\mathrm{pmol} / 1]^{-1}\right)$ & $0.07 \pm 0.01(0.02-0.20)$ & $0.08 \pm 0.01(0.02-0.27)$ & 0.03 \\
\hline IS $_{\text {OGTT }}$ (arbitrary units) & $13.6 \pm 1.1(4.4-32.1)$ & $16.3 \pm 1.2(4.0-40.6)$ & 0.009 \\
\hline
\end{tabular}

Values represent mean \pm SE (range)

${ }^{a} p$ values are for paired differences

Insulin sensitivity clamp data ( $\mathrm{IS}_{\text {clamp }}$ ) at baseline were available in $n=48$

IS $_{\text {clamp }}$ and MRT measurements at follow-up were available in $n=40$ and $n=51$

IS $_{\text {OGTT }}$ OGTT-derived insulin sensitivity; MRT, magnetic resonance tomography 
reflecting activities of these enzymes in the liver are associated with liver fat and insulin sensitivity in humans. The indexes of $\Delta 5$ (20:4 n-6: 20:3n-6) and $\Delta 6$ (18:3n-6: 18:2 n-6) desaturase were not correlated with liver fat or insulin sensitivity ( $p \geq 0.25$ for all).

Since obesity is a strong determinant of high liver fat and insulin resistance, we divided the participants into two groups by the median percentage of body fat (lean, $n=27$, body fat $26.5 \pm 1 \%$; obese, $n=27$, body fat $35.6 \pm 1 \%$ ) and investigated the relationships of hepatic SCD1 activity with liver fat and insulin sensitivity within each group. In lean participants, the hepatic SCD1 activity index, adjusted for sex and body fat, was not correlated with liver fat $(p=0.34)$ or OGTT-derived $(p=0.75)$ or clamp-derived insulin sensitivity ( $p=0.24$; Fig. $1 \mathrm{a}-\mathrm{c})$. In contrast, very strong correlations of the index with liver fat and OGTT- and clamp-derived insulin sensitivity measures were found in obese individuals $(r=-0.57, p=0.004 ; r=0.69, p=0.0002$; $r=0.58, p=0.006$, respectively, Fig. $1 \mathrm{~d}-\mathrm{f}$ ).
Furthermore, we tested the relationships of the SCD1 activity index estimated from the serum NEFA fraction. Under fasting conditions most of the serum NEFA derive from adipose tissue [12]. The adipose tissue SCD1 activity index was positively associated with the hepatic SCD1 index, albeit weakly $(r=0.32, p=0.053)$. In obese participants of this subgroup, moreover, the relationships between the hepatic SCD1 activity index and adjusted liver fat $(p=$ $0.002)$ and insulin sensitivity $(p=0.0002)$ were stronger than those between the adipose tissue SCD1 activity index and the same two parameters ( $p=0.02$ and $p=0.02$, respectively).

\section{Longitudinal data}

Under a lifestyle intervention, there was a significant decrease in BMI, percentage of body fat and visceral fat, as well as a large decrease in liver fat (Table 1). Fasting TG, total and LDL-cholesterol levels, $2 \mathrm{~h}$ insulinaemia and both fasting and $2 \mathrm{~h}$ glycaemia decreased, while insulin
Fig. 1 Cross-sectional relationships at baseline of the hepatic SCD1 activity index with liver fat (a, d), adjusted for sex and body fat, and with insulin sensitivity estimated from the OGTT (b, e) and determined by the euglycaemic-hyperinsulinaemic clamp (c, f), adjusted for age, sex and body fat, in lean (body fat: $26.5 \pm 1 \%)(\mathbf{a}-\mathbf{c})$ and obese (body fat: $35.6 \pm 1 \%$ ) individuals $(\mathbf{d}-\mathbf{f})$. Continuous lines, regression lines; dotted lines, $95 \%$ CIs. $r=0.20, p=0.34$ (a); $r=0.07, p=0.75$ (b); $r=0.26, p=0.24$ (c); $r=0.57$, $p=0.004$ (d); $r=0.69, p=0.0002$ (e); $r=0.58, p=0.006$ (f). $\mathrm{AU}$, arbitary units a

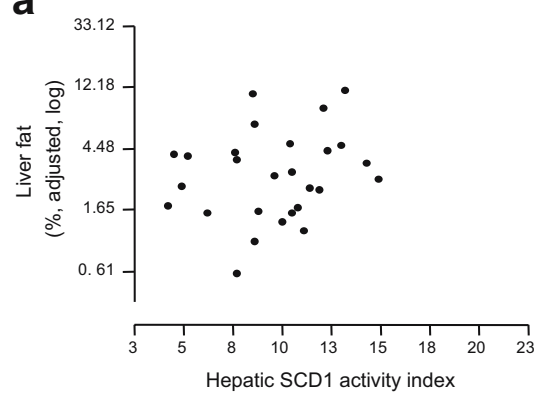

b

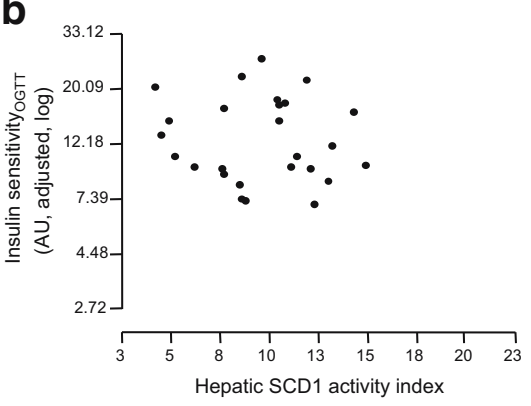

C

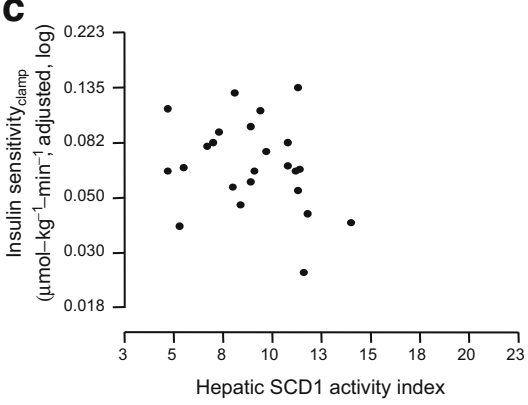

d
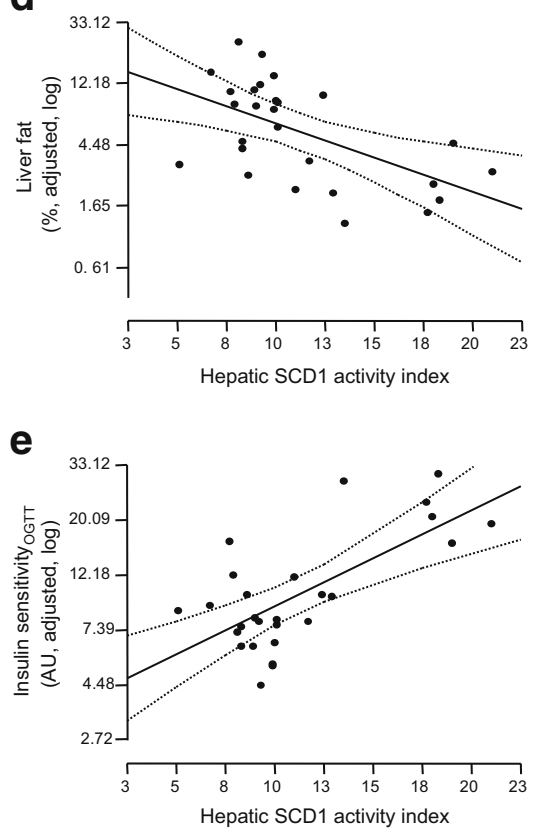

f

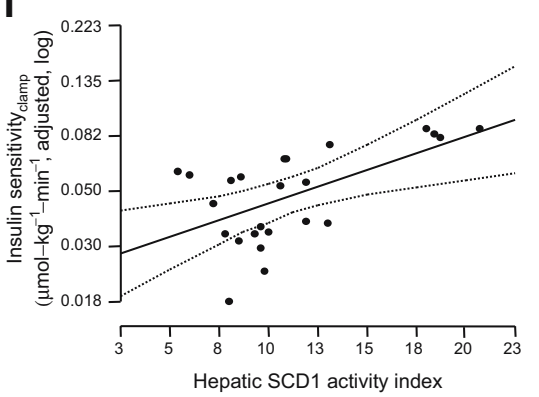


sensitivity increased. Based on the cross-sectional findings between hepatic SCD1 activity and liver fat, we next asked whether the hepatic SCD1 activity index at baseline predicted change in liver fat in prospective analyses. Among parameters at baseline, such as sex, age, total body fat, visceral fat, insulin sensitivity estimated from the OGTT and measured by the clamp, fasting serum NEFA, total cholesterol, LDL- and HDL-cholesterol, TG and the hepatic SCD1 activity index, only sex was significantly associated with change in liver fat in univariate analyses in the lean group (fold-change men $=0.49$, women $=0.94$, $p=0.03$ ). In contrast, only the hepatic SCD1 activity index was significantly associated with change in liver fat in the obese group (Fig. 2).

\section{Discussion}

In the present study, we investigated the role of hepatic SCD1 activity in the pathophysiology of fatty liver and insulin resistance in humans. We found that the hepatic SCD1 activity index, estimated from the fatty acid pattern in serum VLDL-TGs, was negatively associated with liver fat. In contrast, it was not associated with total body fat or visceral fat, which are known determinants of liver fat [1, 7]. Furthermore, the longitudinal data allowed us to determine whether the hepatic SCD1 activity index predicted change in liver fat. A high hepatic SCD1 activity index at baseline was associated with a decrease in liver fat in obese participants. These findings support the hypothesis that hepatic SCD1 activity may regulate liver fat content in humans.

Furthermore, the hepatic SCD1 activity index was also correlated with insulin sensitivity, both that estimated from the OGTT and that determined by the euglycaemichyperinsulinaemic clamp. Interestingly, we found a weak correlation of the hepatic SCD1 activity index with insulin sensitivity determined by the clamp, which is a function of both insulin-stimulated glucose disposal and hepatic insulin sensitivity in the suppression of endogenous glucose production. In contrast, the hepatic SCD1 activity index correlated more strongly with insulin sensitivity estimated from the OGTT and derived from fasting values such as the homeostasis model insulin resistance index $(p=0.04$, data not shown). The latter two measurements closely represent hepatic insulin resistance $[1,37]$. Because of the relationship of hepatic SCD1 activity with liver fat and since fatty liver is closely associated with hepatic insulin resistance $[2,5,7]$, these data support the hypothesis that SCD1 may be involved in the regulation of insulin sensitivity via regulation of fat accumulation in the liver. In addition, since high liver fat also induces hepatic, as well as skeletal muscle insulin resistance via increased production of fetuinA [38], hepatic SCD1 activity may also be involved in the regulation of skeletal muscle insulin sensitivity. In support of this hypothesis, we found a negative correlation between hepatic SCD1 activity and circulating fetuin-A in obese participants $(r=-0.53, p=0.027)$. The hepatic indexes of the $\Delta 5$ and $\Delta 6$ desaturases did not display such correlations with liver fat or insulin sensitivity, suggesting that the activities of these enzymes in the liver are less important for the regulation of liver fat and insulin sensitivity in humans.

Because fat in the liver commonly accumulates and insulin sensitivity declines with increasing adiposity, we tested whether the observed correlations depended on adiposity. In lean participants, no correlations were found between hepatic SCD1 activity and both liver fat and insulin sensitivity, whereas very strong correlations were seen in obese participants. There are several possible explanations for these differences. It may be that SCD1 activity becomes important in the regulation of liver fat and insulin sensitivity as adiposity increases. This hypothesis is supported by recent findings that SCD1 activity is upregulated in obesity. Warensjo et al. [23] found that SCD1 activity in serum cholesteryl esters under fasting conditions in humans predicted an increased risk of developing the metabolic syndrome. However, since this association was not independent of obesity and due to the fact that diets with a high amount of saturated fatty acids [36] and glucose [39] increased SCD1 activity, the authors of that report concluded that the observed relationships reflected compensatory upregulation of SCD1 activity to continuous intake of unhealthy diets [23]. Furthermore, in L6 myotubes
Fig. 2 Relationships of the hepatic SCD1 activity index at baseline with fold-change (baseline to follow-up) in liver fat in lean (body fat: $26.5 \pm 1 \%$ ) (a) and obese (body fat: $35.6 \pm$ $1 \%)$ (b) participants. Continuous lines, regression lines; dotted lines, 95\% CIs; dashed lines, no change in liver fat. $r=0.03, p=$ 0.87 (a); $r=-0.46, p=0.02$ (b)
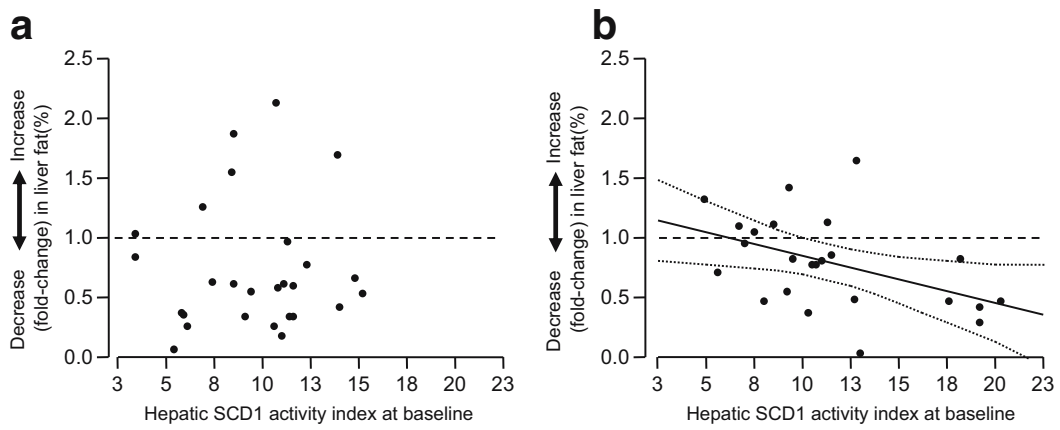
SCD1 overproduction does not improve insulin signalling in basal conditions, but does protect from fatty acid-induced insulin resistance [40]. These data, together with our findings in humans, suggest that SCD1 may particularly protect from obesity-induced insulin resistance.

In the present study, we estimated hepatic SCD1 activity from the fatty acid pattern within the VLDL-TG fraction. VLDL-TG are synthesised in the liver from fatty acids originating from adipose tissue lipolysis $(62 \%)$, intrahepatic de novo lipogenesis (23\%) and dietary lipids [12]. Under fasting conditions, serum NEFA predominantly arise from adipose tissue lipolysis (82\%) [12]. The SCD1 activity index estimated from the fatty acid pattern within the NEFA fraction was only weakly correlated with the hepatic SCD1 activity index, insulin sensitivity and liver fat. Therefore, the SCD1 activity index estimated from VLDL-TG may not only reflect the fatty acid pattern and SCD1 activity within the adipose tissue, but also hepatic SCD1 activity.

The role of SCD1 activity in lipid and glucose metabolism has been extensively studied in vitro and in rodents, and SCD1 has been identified as a potential drug target to improve insulin sensitivity [41]. Mice lacking whole-body SCD1 were lean, were protected against adiposity [24] and displayed increased insulin signalling in skeletal muscle [42]. They also had decreased liver fat content, suggesting that SCD1 deficiency protected against fatty liver $[25,26]$. Interestingly, however, these findings in SCD1-deficient animals were mostly associated with a lean phenotype. Hepatic steatosis can develop in a dietary mouse model despite sustained downregulation of SCD1 [43]. In addition, total SCD1 deficiency resulted in a noticeable phenotype with alopecia [44], abnormalities in the epidermal lipid barrier leading to breakdown of thermoregulation and a metabolic wasting syndrome that could in part explain the excessive energy expenditure [45]. Moreover, SCD1deficient mice on a low-fat, high-carbohydrate diet developed severe hypercholesterolaemia and had a large decrease in HDL-cholesterol levels [27]. In this respect it is also important to mention that loss of hepatic SCD1 activity leads to significantly lower VLDL-TG content in vivo and TG secretion in isolated hepatocytes $[18,46]$. Furthermore, hepatic SCD1 and diacylglycerol acyltransferase closely interact for the final step of TG synthesis and control their composition and subsequent secretion as VLDL [47]. Thus, the latter findings suggest that liver-specific downregulation of SCD1 may impair VLDL assembly and subsequently may reduce the capacity of the liver to clear intrahepatic TG. This hypothesis was supported by recent data showing that reduction of hepatic SCD1 expression by antisense oligonucleotides did not result in a decrease, but in an increase in hepatic lipid content in rodents [28]. In addition, very recently and in contrast to whole-body SCD1 deficiency $[25,26]$, hepatic-specific SCD1 deficiency did not protect from high-fat diet-induced hepatic steatosis or insulin resistance [29]. Furthermore, it was shown that: (1) genetically obese $(o b / o b)$ whole-body SCD1-deficient mice displayed an accelerated progression to severe diabetes [48]; (2) high SCD1 activity protected both from obesity and from palmitate-induced insulin resistance and lipotoxicity [40, 49]; and (3) the insulin-sensitisers thiazolidinediones increased the expression of $S C D 1$ mRNA in adipose tissue and this increased expression of $S C D 1$ was associated with increased insulin sensitivity [50]. Therefore, it is necessary to precisely test the effects of more moderate regulation of SCD1 activity on metabolism, particularly in the light of SCD1 having become an important drug target.

In conclusion, with this approach we provide novel data that a high hepatic SCD1 activity index is associated with low liver fat content and high insulin sensitivity in obese participants. These data suggest that high hepatic SCD1 activity may protect from fat accumulation in the liver and insulin resistance in obesity.

Acknowledgements The authors would like to thank all participants for their cooperation. The study was supported by a grant from the Deutsche Forschungsgemeinschaft (KFO 114), the European Community's FP6 EUGENE2 (LSHM-CT-2004-512013) and the Ministry of Education, Youth and Sport of the Czech Republic (MSM0021627502).

Duality of interest The authors declare that there is no duality of interest associated with this manuscript.

\section{References}

1. Angulo P (2002) Nonalcoholic fatty liver disease. N Engl J Med 346:1221-1231

2. Kelley DE, McKolanis TM, Hegazi RA, Kuller LH, Kalhan SC (2003) Fatty liver in type 2 diabetes mellitus: relation to regional adiposity, fatty acids, and insulin resistance. Am J Physiol Endocrinol Metab 285:E906-E916

3. Marchesini G, Babini M (2006) Nonalcoholic fatty liver disease and the metabolic syndrome. Minerva Cardioangiol 54:229-239

4. Petersen KF, Dufour S, Feng J et al (2006) Increased prevalence of insulin resistance and nonalcoholic fatty liver disease in AsianIndian men. Proc Natl Acad Sci U S A 103:18273-18277

5. Roden M (2006) Mechanisms of disease: hepatic steatosis in type 2 diabetes - pathogenesis and clinical relevance. Nat Clin Pract Endocrinol Metab 2:335-348

6. Targher G, Bertolini L, Poli F et al (2005) Nonalcoholic fatty liver disease and risk of future cardiovascular events among type 2 diabetic patients. Diabetes 54:3541-3546

7. Utzschneider KM, Kahn SE (2006) Review: The role of insulin resistance in nonalcoholic fatty liver disease. J Clin Endocrinol Metab 91:4753-4761

8. Bugianesi E, Pagotto U, Manini R et al (2005) Plasma adiponectin in nonalcoholic fatty liver is related to hepatic insulin resistance and hepatic fat content, not to liver disease severity. J Clin Endocrinol Metab 90:3498-3504

9. Kantartzis K, Rittig K, Balletshofer B et al (2006) The relationships of plasma adiponectin with a favorable lipid profile, 
decreased inflammation, and less ectopic fat accumulation depend on adiposity. Clin Chem 52:1934-1942

10. Stefan N, Machicao F, Staiger H et al (2005) Polymorphisms in the gene encoding adiponectin receptor 1 are associated with insulin resistance and high liver fat. Diabetologia 48:2282-2291

11. Kantartzis K, Fritsche A, Machicao F et al (2007) Upstream transcription factor 1 gene polymorphisms are associated with high antilipolytic insulin sensitivity and show gene-gene interactions. J Mol Med 85:55-61

12. Donnelly KL, Smith CI, Schwarzenberg SJ, Jessurun J, Boldt MD, Parks EJ (2005) Sources of fatty acids stored in liver and secreted via lipoproteins in patients with nonalcoholic fatty liver disease. J Clin Invest 115:1343-1351

13. Westerbacka J, Lammi K, Hakkinen AM et al (2005) Dietary fat content modifies liver fat in overweight nondiabetic subjects. J Clin Endocrinol Metab 90:2804-2809

14. Tiikkainen M, Bergholm R, Vehkavaara S et al (2003) Effects of identical weight loss on body composition and features of insulin resistance in obese women with high and low liver fat content. Diabetes 52:701-707

15. Hudgins LC, Hellerstein M, Seidman C, Neese R, Diakun J, Hirsch J (1996) Human fatty acid synthesis is stimulated by a eucaloric low fat, high carbohydrate diet. J Clin Invest 97:20812091

16. Ueki K, Kadowaki T, Kahn CR (2005) Role of suppressors of cytokine signaling SOCS-1 and SOCS-3 in hepatic steatosis and the metabolic syndrome. Hepatol Res 33:185-192

17. Taskinen MR (2003) Diabetic dyslipidaemia: from basic research to clinical practice. Diabetologia 46:733-749

18. Miyazaki M, Kim YC, Ntambi JM (2001) A lipogenic diet in mice with a disruption of the stearoyl-CoA desaturase 1 gene reveals a stringent requirement of endogenous monounsaturated fatty acids for triglyceride synthesis. J Lipid Res 42:1018-1024

19. Jones BH, Maher MA, Banz WJ et al (1996) Adipose tissue stearoylCoA desaturase mRNA is increased by obesity and decreased by polyunsaturated fatty acids. Am J Physiol 271:E44-E49

20. Pan DA, Lillioja S, Milner MR et al (1995) Skeletal muscle membrane lipid composition is related to adiposity and insulin action. J Clin Invest 96:2802-2808

21. Storlien LH, Jenkins AB, Chisholm DJ, Pascoe WS, Khouri S, Kraegen EW (1991) Influence of dietary fat composition on development of insulin resistance in rats. Relationship to muscle triglyceride and omega-3 fatty acids in muscle phospholipid. Diabetes 40:280-289

22. Vessby B, Aro A, Skarfors E, Berglund L, Salminen I, Lithell H (1994) The risk to develop NIDDM is related to the fatty acid composition of the serum cholesterol esters. Diabetes 43:1353-1357

23. Warensjo E, Riserus U, Vessby B (2005) Fatty acid composition of serum lipids predicts the development of the metabolic syndrome in men. Diabetologia 48:1999-2005

24. Ntambi JM, Miyazaki M, Stoehr JP et al (2002) Loss of stearoylCoA desaturase-1 function protects mice against adiposity. Proc Natl Acad Sci U S A 99:11482-11486

25. Cohen P, Miyazaki M, Socci ND et al (2002) Role for stearoylCoA desaturase-1 in leptin-mediated weight loss. Science 297: 240-243

26. Dobrzyn P, Dobrzyn A, Miyazaki M et al (2004) Stearoyl-CoA desaturase 1 deficiency increases fatty acid oxidation by activating AMP-activated protein kinase in liver. Proc Natl Acad Sci U S A 101:6409-6414

27. Flowers MT, Groen AK, Oler AT et al (2006) Cholestasis and hypercholesterolemia in SCD1-deficient mice fed a low-fat, highcarbohydrate diet. J Lipid Res 47:2668-2680

28. Gutierrez-Juarez R, Pocai A, Mulas C et al (2006) Critical role of stearoyl-CoA desaturase-1 (SCD1) in the onset of diet-induced hepatic insulin resistance. J Clin Invest 116:1686-1695
29. Miyazaki M, Flowers MT, Sampath H et al (2007) Hepatic stearoyl-CoA desaturase-1 deficiency protects mice from carbohydrate-induced adiposity and hepatic steatosis. Cell Metab 6:484-496

30. Machann J, Thamer C, Schnoedt B et al (2005) Standardized assessment of whole body adipose tissue topography by MRI. J Magn Reson Imaging 21:455-462

31. Szczepaniak LS, Nurenberg P, Leonard D et al (2005) Magnetic resonance spectroscopy to measure hepatic triglyceride content: prevalence of hepatic steatosis in the general population. Am J Physiol Endocrinol Metab 288:E462-E468

32. Expert Committee on the Diagnosis and Classification of Diabetes Mellitus (1997) Report of the Expert Committee on the diagnosis and classification of diabetes mellitus. Diabetes Care 20:11831197

33. Matsuda M, DeFronzo RA (1999) Insulin sensitivity indices obtained from oral glucose tolerance testing: comparison with the euglycemic insulin clamp. Diabetes Care 22:1462-1470

34. Stefan N, Wahl HG, Fritsche A, Haring H, Stumvoll M (2001) Effect of the pattern of elevated free fatty acids on insulin sensitivity and insulin secretion in healthy humans. Horm Metab Res 33:432-438

35. Kim YC, Gomez FE, Fox BG, Ntambi JM (2000) Differential regulation of the stearoyl-CoA desaturase genes by thiazolidinediones in 3T3-L1 adipocytes. J Lipid Res 41:1310-1316

36. Vessby B, Gustafsson IB, Tengblad S, Boberg M, Andersson A (2002) Desaturation and elongation of fatty acids and insulin action. Ann NY Acad Sci 967:183-195

37. Meyer C, Pimenta W, Woerle HJ et al (2006) Different mechanisms for impaired fasting glucose and impaired postprandial glucose tolerance in humans. Diabetes Care 29:1909-1914

38. Stefan N, Hennige AM, Staiger H et al (2006) Alpha2-HeremansSchmid glycoprotein/fetuin-A is associated with insulin resistance and fat accumulation in the liver in humans. Diabetes Care $29: 853-857$

39. Houdali B, Wahl HG, Kresi M et al (2003) Glucose oversupply increases Delta9-desaturase expression and its metabolites in rat skeletal muscle. Diabetologia 46:203-212

40. Pinnamaneni SK, Southgate RJ, Febbraio MA, Watt MJ (2006) Stearoyl CoA desaturase 1 is elevated in obesity but protects against fatty acid-induced skeletal muscle insulin resistance in vitro. Diabetologia 49:3027-3037

41. Dobrzyn A, Ntambi JM (2005) Stearoyl-CoA desaturase as a new drug target for obesity treatment. Obes Rev 6:169-174

42. Rahman SM, Dobrzyn A, Dobrzyn P, Lee SH, Miyazaki M, Ntambi JM (2003) Stearoyl-CoA desaturase 1 deficiency elevates insulin-signaling components and down-regulates protein-tyrosine phosphatase 1B in muscle. Proc Natl Acad Sci U S A 100:11110 11115

43. Toye AA, Dumas ME, Blancher C et al (2007) Subtle metabolic and liver gene transcriptional changes underlie diet-induced fatty liver susceptibility in insulin-resistant mice. Diabetologia 50: $1867-1879$

44. Miyazaki M, Dobrzyn A, Elias PM, Ntambi JM (2005) StearoylCoA desaturase-2 gene expression is required for lipid synthesis during early skin and liver development. Proc Natl Acad Sci U S A 102:12501-12506

45. Binczek E, Jenke B, Holz B, Gunter RH, Thevis M, Stoffel W (2007) Obesity resistance of the stearoyl-CoA desaturase-deficient (scd1-/-) mouse results from disruption of the epidermal lipid barrier and adaptive thermoregulation. Biol Chem 388:405-418

46. Legrand P, Catheline D, Fichot MC, Lemarchal P (1997) Inhibiting delta9-desaturase activity impairs triacylglycerol secretion in cultured chicken hepatocytes. J Nutr 127:249-256

47. Man WC, Miyazaki M, Chu K, Ntambi J (2006) Colocalization of SCD1 and DGAT2: implying preference for endogenous mono- 
unsaturated fatty acids in triglyceride synthesis. J Lipid Res 47:1928-1939

48. Flowers JB, Rabaglia ME, Schueler KL et al (2007) Loss of stearoylCoA desaturase-1 improves insulin sensitivity in lean mice but worsens diabetes in leptin-deficient obese mice. Diabetes 56:1228-1239

49. Busch AK, Gurisik E, Cordery DV et al (2005) Increased fatty acid desaturation and enhanced expression of stearoyl coenzyme
A desaturase protects pancreatic beta-cells from lipoapoptosis. Diabetes 54:2917-2924

50. Riserus U, Tan GD, Fielding BA et al (2005) Rosiglitazone increases indexes of stearoyl-CoA desaturase activity in humans: link to insulin sensitization and the role of dominant-negative mutation in peroxisome proliferator-activated receptor-gamma. Diabetes 54:1379-1384 